\title{
DIMINUIÇÃO DO ÍNDICE DE PERDAS DE MATÉRIA-PRIMA COM APLICAÇÃO DE INDICADORES DE PROCESSOS: PLANO DE CORTE BIDIMENSIONAL PARA BLINDAGEM AUTOMOBILÍSTICA
}

\author{
Paula Christina Braulio da Costa (UNIGRANRIO) paulabraulio@unigranrio.br \\ Beatriz Inacio Silva da Fonseca (UNIGRANRIO) beatriz.fonseca.inacio@gmail.com \\ Marcos dos Santos (Instituto Militar de Engenharia) marcosdossantos_doutorado_uff@yahoo.com.br \\ Maria Cristina Ravagnani (UNIGRANRIO) crisravagnani@unigranrio.edu.br \\ Rubens Aguiar Walker (UNIGRANRIO) rubens.walker@unigranrio.edu.br
}

\section{Resumo}

O presente estudo tem como objetivo a busca de melhorias para a diminuição do desperdício e perda de matéria prima de um processo de corte em uma empresa de blindagem automobilística. Inicialmente, foram realizadas pesquisas bibliográficas para o levantamento de informações referentes ao Business Process Management e aos Indicadores de Processo, para a melhor compreensão dos mesmos. Posteriormente, realizou-se o estudo na empresa de nome fictício PCB, dando inicio a coleta de dados necessários para a aplicação do método, através dos indicadores de processos, mapeamento de processo, ferramentas da qualidade e do Software Powernest, procurou-se priorizar as causas nos pontos críticos do processo produtivo que apresentavam perda de matéria prima.

Atualmente o mercado se mantém em constante busca por melhores práticas de qualidade nas empresas, e a sociedade em geral busca praticidade aliada à segurança ao adquirir um serviço de blindagem, baseada nesta proposta, quando na empresa faz-se o melhor aproveitamento da matéria-prima, a sociedade tem como beneficio a redução no custo de aquisição do automóvel blindado e a empresa alcança melhorias em sua produtividade e qualidade no processo, de modo que a mantenha competitiva no mercado. A ação de melhoria aplicada neste estudo de caso alcançou seu objetivo de reduzir o índice de perda e assim otimizar $80 \%$ o processo de corte, consequentemente a otimização do processo, o aumento da produtividade e lucro da empresa.

Palavras-Chaves: Business Process Management (BPM), Produtividade, Perda de Matéria Prima, Indicadores de Processo, Mapeamento de Processos. 


\section{Introdução}

A Grande Revolução Industrial ocorrida no século XIX modificou o cenário mundial, obrigando as indústrias a procurar excelência diante de um mercado cada vez mais competitivo e inovador, essa busca com o passar dos anos trouxe modificações a todos os ramos e setores industriais, atualmente uma das principais preocupações dos administradores e pesquisadores são as áreas de produção e logística. Os clientes são a meta e o foco principal da competição por excelência e a busca por processos que gerem eficiência e qualidade em seus produtos, operações e serviços prestados.

De acordo com Campos (2010), só é possível ter total controle sobre os processos tendo, padrões planejados e bem projetados e operadores treinados e conscientizados das metas que devem ser atingidas em cada setor de operação.

Segundo Milan (2012), a gestão de processos procura entregar resultados que tenham valor para o cliente, sendo eles produtos ou serviços, organizando uma série de atividades transacionais da empresa. As atividades constituem um processo que recebem uma entrada, adicionam valor com um processo de transformação e entregam uma saída ao cliente, estando diretamente relacionadas à coordenação do fluxo de objetos da empresa.

De acordo com a ABPMP (2013, p.1), o BPM - Business Process Modeler ou gerenciamento de processos de negócios é um novo modelo de aplicar de forma integrada abordagens, metodologias, estruturas, práticas, técnicas e ferramentas para processos que na grande maioria são aplicados de maneira isolada. Sendo assim, o BPM é uma visão holística de organização e estruturação de como conduzir o negócio, reconhecendo o papel-chave de pessoas com habilidade e motivação para os processos de negócio, assim como o uso correto de tecnologias para entregar melhores produtos e serviços para os clientes.

O mesmo se baseia em um conjunto de métodos, técnicas e ferramentas de apoio ao projeto, à criação, ao controle e à análise dos processos, a partir da gestão de estratégias, e consequentemente da definição de seus indicadores de processo (MILNITZ, 2016).

A definição de indicador de desempenho de processo está associada a um padrão e uma variável aleatória em função do tempo. Então, definem-se indicadores como meios quantificáveis, podendo ser classificado como indicador de características: atributos ou requisitos; de produtos: bens e serviços ou de processos: conjunto de causas e condições que transforma recursos em produtos, utilizados para acompanhar e melhorar os resultados ao longo do tempo (ZANIN, 2015). 
A medição de um índice obtido por um indicador leva somente ao conhecimento dos fenômenos reais que estão ocorrendo no processo, controlá-lo significa manter o mesmo estável diante das ocorrências. Esse equilíbrio envolve distintas técnicas estatísticas e ferramentas da qualidade para obter o controle esperado do processo. Estas ferramentas são aplicadas para que seja possível localizar os problemas, analisar e eliminar as causas, estabelecendo padrões e controles (GOULART, 2010).

A melhoria só é obtida através de alguma metodologia específica, sendo assim, este estudo pretende aplicar os indicadores de processo, com o auxilio BPM e as ferramentas da qualidade no processo de corte das placas para blindagem de carros, a fim de trazer melhorias no processo, eliminação de perdas da matéria prima e padronização do processo na empresa de nome fictício PCB.

\section{Descrição do Problema}

A empresa PCB é do ramo automobilístico, fundada com o propósito de superar o que é esperado dos serviços de blindagem no Brasil. Uma análise no processo de corte foi elaborada, identificando que havia desperdício de matéria prima. O profissional responsável pela elaboração dos moldes e utilização da matéria-prima, que são a Manta de Aramida e a Placa de Aço, possuindo pouco conhecimento na utilização desse material, ao fazer o corte ocasionava grande perda do mesmo.

O gráfico abaixo apresenta o total gasto com matéria prima no período de um ano, na amostragem temos o total de 1000 placas utilizadas para a blindagem de 100 carros Toyota Corolla, onde cada carro blindado utiliza 10 placas, sendo 5 placas $\left(8 \mathrm{~m}^{2}\right)$ da Manta de Aramida que custa em torno de $\mathrm{R} \$ 790,00$ reais o $\mathrm{m}^{2}$ e 5 placas da Placa de Aço $\left(8 \mathrm{~m}^{2}\right)$, que custa em torno $\mathrm{R} \$ 1.438,00{\mathrm{o} \mathrm{m}^{2}}^{2}$ reais cada. 
Gráfico 1- Custo Anual de Matéria Prima

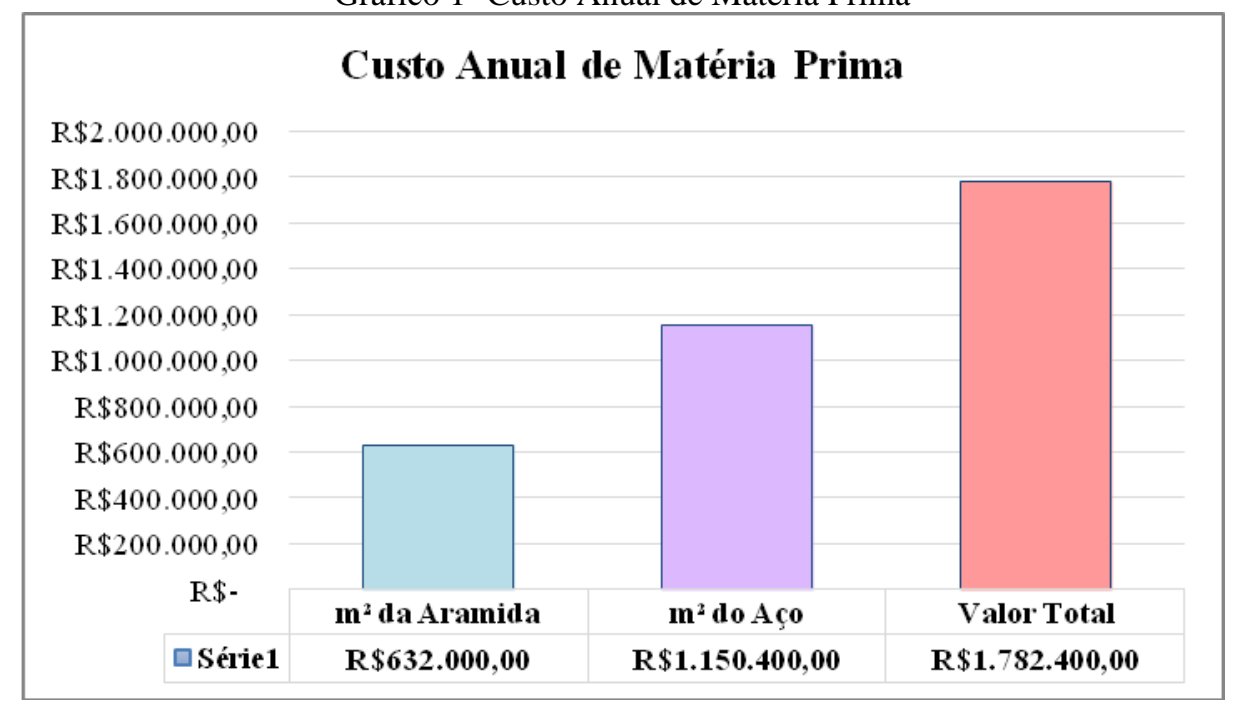

Fonte: Autores (2019)

Em função das perdas de matéria-prima ocorridas, necessário se fez buscar meios para otimização e melhoria do processo, visando à padronização do mesmo. Para isso serão utilizados indicadores de processo, entre eles, ferramentas da qualidade, mapeamento de processo e por fim um software de otimização.

$\mathrm{Na}$ busca da resposta para o problema da empresa PCB, a pergunta a ser respondida; Como reduzir perdas de matéria-prima e ao mesmo tempo otimizar o processo de corte?

\section{Procedimentos Metodológicos}

\subsection{BPM - Business Process Management}

De acordo com a ABPMP (2009), um dos principais modelos de gerenciar os processos de negócio é por meio do ciclo de vida do BPM, que pode ser contextualizado como um conjunto interativo de atividades que incluem: planejamento, análise, desenho e modelagem, implantação, monitoramento e controle dos processos de negócio. O BPM tem o intuito de auxiliar na tomada de decisão, permitindo que gerentes e executivos possam se dedicar mais às decisões importantes, de maneira que ajudem na solução de diversos problemas e facilitam o entendimento do funcionamento das atividades complexas diminuindo o tempo ou desperdícios desprendidos ao final do processo. (OLIVEIRA et. al. 2018).

De acordo com a ABPMP (2009), a solução para melhoria de processos tem como objetivo obter mais resultado utilizando menos recursos, onde estão diretamente associadas ao nível de maturidade que os processos possuem, sendo desde o estado inicial, repetível, definido, 
gerenciado até o otimizado. Constantemente, estas melhorias são proporcionadas por ações relacionadas a pessoas, tecnologias, processos, cultura e desempenho, entre outros.

\subsection{Indicadores de Processo}

Na visão de Martins (2004), o uso de indicadores de desempenho de processo, relaciona-se a entrega de valor ao cliente e com o desempenho global da organização, alinhando e permitindo melhorar os objetivos de todos que são afetados pelos negócios. O autor (2004) conceitua que, os indicadores são ferramentas principais por permitirem um acompanhamento das essenciais variáveis de interesse da organização e por proporcionar o planejamento de ações tendo em vista melhorias de desempenho.

De acordo com Bortoluzzi (2011), os indicadores de desempenho de processo são uma ferramenta de gestão utilizada nas distintas áreas empresariais para a medição do desempenho de sucesso em uma determinada área, processo ou operação. Com sua utilização, é possível visualizar o que está funcionando ou não dentro de uma empresa e qual seu real desempenho, e ainda assim permite a colaboração de todos que trabalham no processo e na empresa.

\subsection{Mapeamento de Processo}

De acordo com Brocke et. al. (2014), o surgimento de organizações guiadas por processos pode ser um ponto fundamental na administração de empresas, causando uma mudança no paradigma das organizações.

Anteriormente, o foco era na estruturação funcional, no qual gerava uma grande especialização dos colaboradores e uma visão parcial do funcionamento de toda organização, ocasionando conflitos. Por meio disso, o mapeamento de processos é definido como uma organização específica das atividades de trabalho, com um começo, um fim e entradas e saídas identificadas.

Na visão de Cunha (2012), a modelagem de processos deve ser executada sempre que ocorrerem oportunidades de melhoria identificadas, aprimorando os pontos fortes do processo. O resultado após o mapeamento tende a expor as alternativas de solução a problemas mapeados.

\subsection{Ferramentas da Qualidade}

As ferramentas da qualidade são técnicas estatísticas e gerencias que auxiliam na obtenção, organização e analises das informações, necessárias para resolução de problemas, utilizando dados quantitativos, são ferramentas de fácil utilização, podendo ser usadas por qualquer 
funcionário da organização, facilitando a implantação da cultura de melhoria continua em todos os níveis operacionais e organizacionais (FONSECA, 2018).

Entende-se que para analisarmos algum problema, estabelecer um sistema de gerenciamento, haverá a necessidade de adotar alguma ferramenta da qualidade, que atenda as necessidades da empresa, para o problema em questão, foram utilizadas algumas ferramentas, como o Gráfico de Pareto, Diagrama de Ishikawa, Brainstorming, 5W2H, Folha de Verificação.

\subsection{Software POWERNEST}

O Powernest é considerado segundo Alma (2017), um software muito eficaz, em projetos para desenvolvimentos voltados para aplicações de melhorias para cortes bidimensionais. O software pode ser utilizado em diversos setores como: corte em chapas metálicas, indústrias de embalagem, estaleiros, corte de plásticos e outros. A utilização desse software dá autonomia e oportunidades de inserção de dados de um estudo piloto e modificar interativamente o layout. O software calcula automaticamente as variações esperadas, o poder estatístico e o design ideal, de maior otimização. O Powernest permite também minimizar a variabilidade, de projetos, deixando tudo conforme o esperado, sem gerar dúvidas no processo final.

\section{Estudo de Caso}

\subsection{Contexto Gerencial da Empresa}

A empresa PCB, consiste em uma organização familiar que atua no setor automobilístico, no ramo de blindagem de carros, fundada em 2010 no estado do Rio de Janeiro. No ano de 2016, tornou-se referência nacional em blindagem de carros. A PCB tem como objetivo diante da violência que cresce de forma progressiva nos grandes centros urbanos estar na vanguarda dos novos conceitos de blindagem automobilística, adquirindo conhecimento e estando sempre a procura de novas ideias para que um número maior de pessoas possam blindar seus carros. Sendo assim, foi desenvolvido um processo eficaz na proteção balística, buscando a excelência e o aperfeiçoamento de seus processos.

O projeto desenvolvido baseou-se no modelo do carro Toyota Corolla, por tratar-se do automóvel com maior número de procura do serviço de blindagem, são blindados em média 100 carros deste modelo por ano, por este motivo foi ele escolhido como objeto de estudo. 


\subsection{Análise do Problema}

A coleta e análise de dados tem como finalidade a priorização do problema, selecionando as causas que influenciam diretamente no processo de corte, ocasionando a perda de matéria prima. Na tabela 1, é apresentado o valor do $\mathrm{m}^{2}$ de cada matéria prima utilizada no processo de corte e blindagem e a tabela 2 dispõe a quantidade de material utilizado por carro e o custo gerado;

Tabela 1 - Custo unitário do material

\begin{tabular}{|l|lc|}
\hline \multicolumn{1}{|c|}{ Matéria Prima } & \multicolumn{2}{c|}{ Custo } \\
\hline $\mathbf{l m}^{\mathbf{2}}$ de Manta de Aramida $(\mathbf{2} \mathbf{m} \mathbf{x} \mathbf{1 , 5 m})$ & RS & 790,00 \\
\hline $\mathbf{l m}^{\mathbf{2}}$ de Placa de Aço (3m $\mathbf{~} \mathbf{1 , 1 0 m )}$ & RS & $1.438,00$ \\
\hline
\end{tabular}

Fonte: Autores (2019)

Tabela 2 - Quantidade de material e custo por carro

\begin{tabular}{|c|c|rr|}
\hline \multicolumn{4}{|c|}{ Quant. Gasta por Carro } \\
\hline $\mathbf{8 m}^{\mathbf{2}}$ de Manta de Aramida & 5 Placas & RS & $6.320,00$ \\
\hline $\mathbf{8 m}^{\mathbf{2}}$ de Placa de Aço & 5 Placas & RS & $11.504,00$ \\
\hline
\end{tabular}

Fonte: Autores (2019)

Por fim, na tabela 3 é apresentada a quantidade $\left(\mathrm{m}^{2}\right)$ e o valor $(\mathrm{R} \$)$ do total de placas de Aramida e de Aço, valores estes obtidos por meio da pesquisa realizada durante o período de um ano, que demonstra dados verídicos da empresa PCB.

Tabela 3 - Custo de Produção Anual

\begin{tabular}{|c|c|c|c|c|c|c|c|c|}
\hline \multicolumn{9}{|c|}{ Tabela de Custo de Produção Anual - Antes do Estudo de Caso } \\
\hline Mês/Ano & Quant. de Carros & Quant. de Placas & & da Manta & & $\mathrm{m}^{2}$ do $A c ̧ o$ & & Talor Total \\
\hline $\mathrm{jul} / 18$ & 6 & 60 & RS & $37.920,00$ & RS & $69.024,00$ & RS & $106.944,00$ \\
\hline ago/18 & 5 & 50 & RS & $31.600,00$ & RS & $57.520,00$ & RS & $89.120,00$ \\
\hline set/18 & 10 & 100 & RS & $63.200,00$ & RS & $115.040,00$ & RS & $178.240,00$ \\
\hline out/18 & 7 & 70 & RS & $44.240,00$ & RS & $80.528,00$ & RS & $124.768,00$ \\
\hline nov/18 & 6 & 60 & $\mathrm{RS}$ & $37.920,00$ & RS & $69.024,00$ & RS & $106.944,00$ \\
\hline dez/18 & 8 & 80 & RS & $50.560,00$ & RS & $92.032,00$ & RS & $142.592,00$ \\
\hline $\mathrm{jan} / 19$ & 15 & 150 & RS & $94.800,00$ & RS & $172.560,00$ & RS & $267.360,00$ \\
\hline fev/19 & 5 & 50 & RS & $31.600,00$ & RS & $57.520,00$ & RS & $89.120,00$ \\
\hline mar/19 & 9 & 90 & $\mathrm{RS}$ & $56.880,00$ & RS & $103.536,00$ & RS & $160.416,00$ \\
\hline abr/19 & 11 & 110 & RS & $69.520,00$ & RS & $126.544,00$ & RS & $196.064,00$ \\
\hline mai/19 & 6 & 60 & RS & $37.920,00$ & RS & $69.024,00$ & RS & $106.944,00$ \\
\hline jun/19 & 8 & 80 & $\mathrm{RS}$ & $50.560,00$ & RS & $92.032,00$ & RS & $142.592,00$ \\
\hline $\mathrm{jul} / 19$ & 4 & 40 & RS & $25.280,00$ & RS & $46.016,00$ & RS & $71.296,00$ \\
\hline Total & 100 & 1000 & RS & $632.000,00$ & RS & $1.150 .400,00$ & RS & $1.782 .400,00$ \\
\hline
\end{tabular}

Fonte: Autores (2019)

Após uma analise do histórico total do processo de corte, no período de julho de 2018 a julho de 2019, a equipe do projeto reuniu-se com o objetivo de identificar as causas potencias dos 
problemas prioritários que ocorriam na empresa, como a perda de material e a falta de padronização do processo.

A partir disso foi elaborado um Brainstorming, com o propósito de visualizar quais os itens que estariam causando maior impacto no processo produtivo, como se observa na figura abaixo.

Figura 1 - Brainstorming do processo de corte

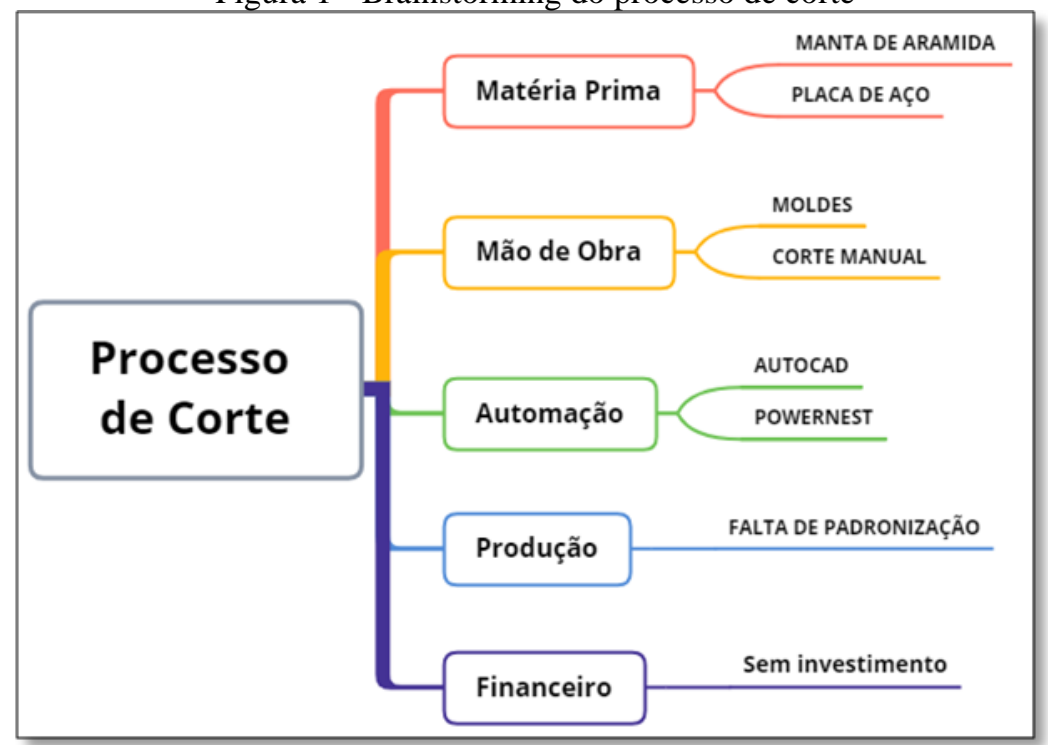

Fonte: Autores (2019)

O Brainstorming elaborado funcionou como uma ferramenta de auxílio na busca das possíveis causas do problema, a partir disso foi elaborado o Diagrama de Ishikawa para seleção das causas potenciais, como pode-se observar na figura 2.

Figura 2 - Diagrama de Ishikawa das possíveis causas de perda no processo de corte

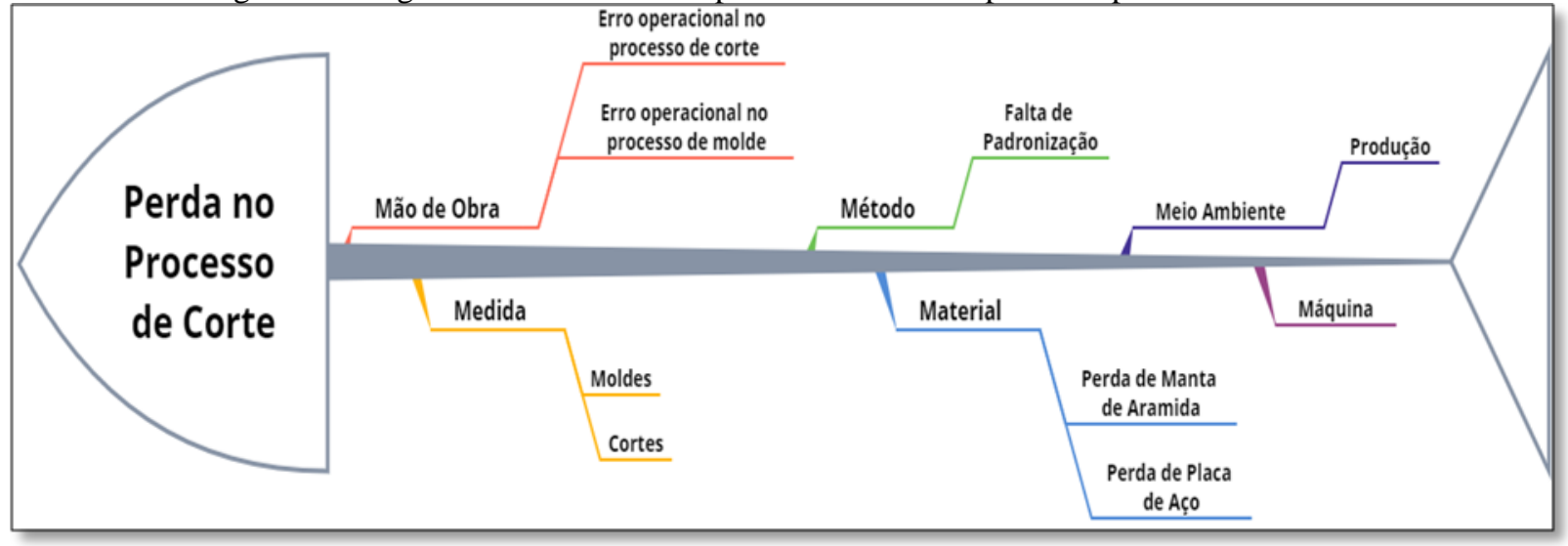

Fonte: Autores (2019)

Foram encontradas oito causas potenciais do problema de perda no processo, após a avaliação definiu-se que os itens primordiais são, o Material, a Mão de Obra, Medida e o Método por 
serem os principais responsáveis pelo desperdício de matéria prima afetando diretamente o processo.

\subsection{Mapeamento do Processo}

Após definir e priorizar as causas optou-se pela elaboração do mapeamento de processo, para identificar onde ocorrem as perdas e o desperdício de material, determinando os pontos críticos com a finalidade da melhoria do processo, com o intuito de aperfeiçoar o desempenho implantando novas estratégias.

Na figura 3, demonstram-se através do mapeamento do processo os pontos críticos;

Figura 3 - Mapeamento do Processo e Pontos Críticos

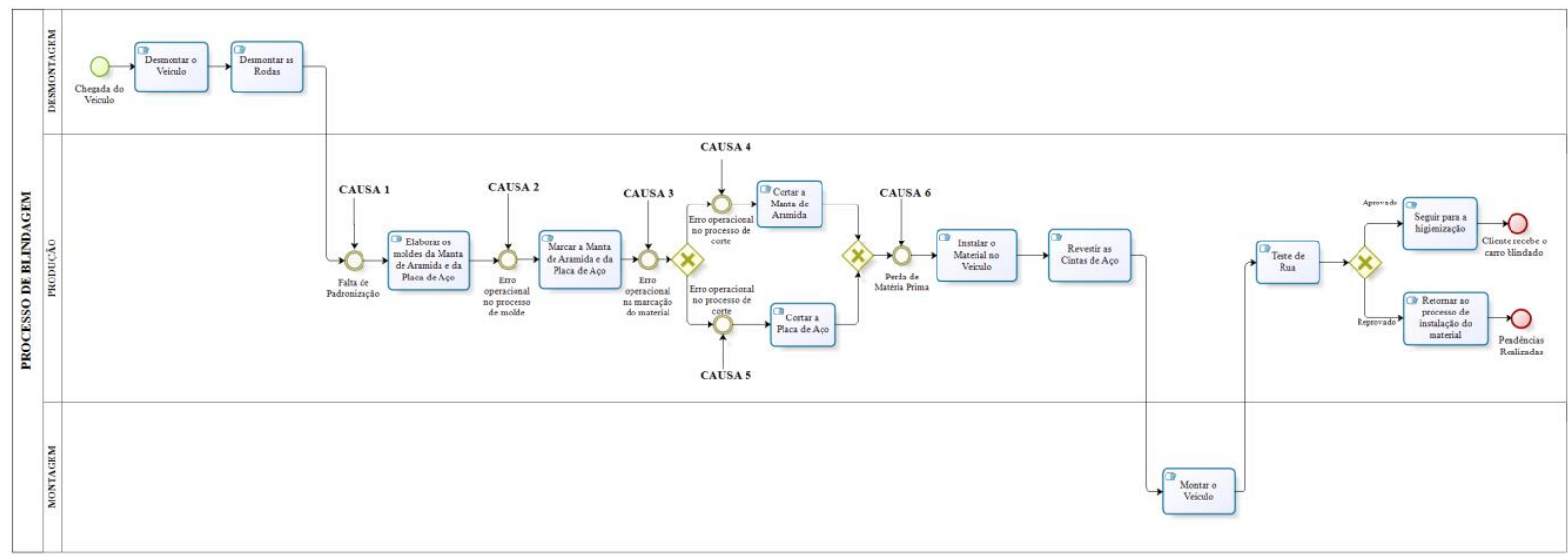

Fonte: Autores (2019)

\subsection{Pontos Críticos do Processo}

Por meio do mapeamento de processo, foi possível identificar em quais etapas do processo encontram-se as causas para as perdas e o desperdício de matéria prima, sendo elas;

- CAUSA 1 - Falta de Padronização: Processo realizado de forma aleatória, causando prejuízo para a empresa, sendo assim, o processo já se inicia com a probabilidade de erros e falhas.

- CAUSA 2 - Erro operacional no processo de molde: Modelagem feita manualmente e de forma aleatória, em função da falta de padronização.

- CAUSA 3 - Erro operacional no processo de marcação: Por ser um processo de fluxo contínuo, a marcação é realizada em função do molde sem padronização. 
- CAUSA 4 - Erro operacional no processo de corte da Manta Aramida: Moldes e Marcações elaborados de formas aleatórias, causadas pelos moldes com medidas erradas.

- CAUSA 5 - Erro operacional no processo de corte da Placa de Aço: Moldes e Marcações elaborados de formas aleatórias, causadas pelos moldes com medidas erradas.

- CAUSA 6 - Perdas de Matéria Prima: Como consequência de todo um processo realizado de forma manual e aleatória.

\subsection{Análise dos pontos críticos do processo}

Com o propósito de analisar as causas prioritárias do problema identificadas através do mapeamento de processo, foi elaborada uma Folha de Verificação anual de Julho/2018 à Julho/2019, apresentada na tabela 4;

Tabela 4 - Consolidado da Folha de Verificação Jul/2018 - Jul/2019

\begin{tabular}{|l|c|}
\hline \multicolumn{2}{|c|}{ Folha de Verificação do Toyota Corolla Consolidado Jul/18 - Jul/19 } \\
\hline Erro operacional no processo de corte & 48 \\
\hline Erro operacional no processo de molde & 48 \\
\hline Falta de padronização & 69 \\
\hline Erro operacional no processo de marcação & 23 \\
\hline Perda de manta aramida & 92 \\
\hline Perda de placa aço & 92 \\
\hline Outras causas & 16 \\
\hline TOTAL & $\mathbf{3 8 3}$ \\
\hline
\end{tabular}

Fonte: Autores (2019)

Por meio da análise da Folha de Verificação, foram adquiridos valores que possibilitaram a elaboração do Gráfico de Pareto, onde foi utilizado para identificar quais os pontos que devem ser melhorados, definir o plano de ação a ser utilizado e qual problema deve ser priorizado, onde a perda de matéria prima é a principal causa a ser priorizada.

No Gráfico 2, é apresentado o Pareto com as causas prioritárias que foram identificadas no mapeamento de processo; 


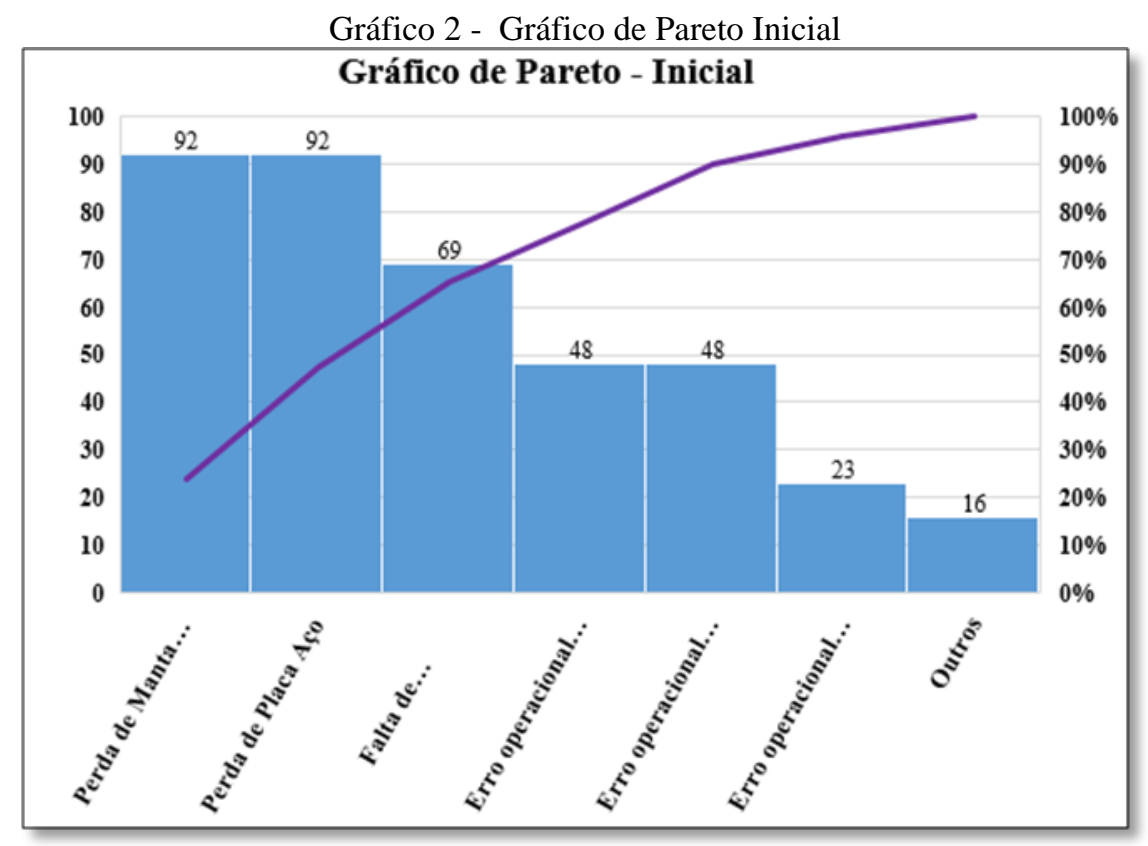

Fonte: Autores (2019)

\subsection{Proposta de Solução}

Em função das análises realizadas e consequente identificação das causas do problema, optouse pela realização de um plano de ação, com a finalidade de eliminar os erros ocorridos durante as etapas do processo de blindagem dos automóveis. Foi elaborado o quadro para apresentar o plano de ação visando a diminuição de perdas no processo. 
Quadro 1 - Plano de Ação Diminuição dos Desperdícios de matéria prima

\begin{tabular}{|c|c|}
\hline \multicolumn{2}{|c|}{$\begin{array}{c}\text { Plano de Ação } \\
\text { Diminuição de Desperdícios } \\
\text { de Matéria-Prima }\end{array}$} \\
\hline What? (O quê?) & $\begin{array}{l}\text { Redução dos desperdicios nas várias etapas do } \\
\text { processo de blindagem automotiva. }\end{array}$ \\
\hline Why? (Por quê?) & $\begin{array}{l}\text { Porque os diversos erros, que ocorrem } \\
\text { principalmente nas etapas de modelagem, marcação } \\
\text { e cortes do material, geram prejuizos com a perda de } \\
\text { matéria prima, para a empresa. }\end{array}$ \\
\hline Where? (Onde?) & $\begin{array}{l}\text { No decorrer de todo processo de blindagem, setor de } \\
\text { produção. }\end{array}$ \\
\hline When? (Quando?) & A partir de Agosto de 2019. \\
\hline Who? (Quem?) & O engenheiro de produção da empresa PCB. \\
\hline How? (Como?) & $\begin{array}{l}\text { Automatizar o processo através do software } \\
\text { Powernest, enviando ao supervisor o gabarito com } \\
\text { com os moldes otimizados, facilitando aos } \\
\text { operadores a marcação direta e correta na manta de } \\
\text { aramida e na placa de aço. } \\
\text { Eliminando a etapa do molde. }\end{array}$ \\
\hline How much? (Quanto?) & Sem investimento monetário. \\
\hline
\end{tabular}

Fonte: Autores (2019)

Após a elaboração do plano de ação, os moldes já utilizados na marcação e corte do material, criados através do programa AutoCAD, foram inseridos no software Powernest, passando por uma remodelagem e assim aperfeiçoou-se o processo.

\subsection{Resultados Alcançados}

Uma vez que, não se tinha uma padronização no processo produtivo na parte operacional de molde, marcação e corte da manta de aramida e da placa de aço, após a implantação do software Powernest, passou-se a ter uma dimensão real das causas que originavam o problema das perdas e desperdícios, com o mesmo obteve-se a padronização desejada no referido processo, eliminando a etapa de molde, de forma que; 
I. O "gabarito" das medidas para elaboração dos moldes do carro x, feito no Autocad é otimizado no software Powernest ajustado as medidas da manta de aramida e da placa de aço.

II. A etapa do molde foi assim automatizada, tendo um padrão para cada tipo de carro, e como isso se diminui o número de placas de aço e manta de aramida utilizada no processo.

\subsection{Padronização do Corte}

A etapa de corte passa a ser realizado sem retrabalho e perda de matéria prima, devido aos novos moldes já automatizados, com isso o desperdício de matéria prima se tornou mínimo, tendo assim um aproveitamento em torno de $80 \%$. Na tabela 5 , pode-se observar os resultados obtidos com a aplicação do Software;

Tabela 5 - Resultados da otimização do Powernest

\begin{tabular}{|c|c|c|c|}
\hline \multicolumn{4}{|c|}{ Moldes Manta Aramida e Placa de Aço } \\
\hline Placas Utilizadas & $\mathbf{3}$ & Cortes & $\mathbf{3 2}$ \\
\hline Peças Produzidas & $\mathbf{3 2}$ & Acabamentos & $\mathbf{0 , 0 0} \mathbf{~ m}$ \\
\hline Retalhos Gerados & $\mathbf{0}$ & Aproveitamento & $\mathbf{8 6 , 4 \%}$ \\
\hline
\end{tabular}

Fonte: Autores (2019)

Os moldes apresentados nestas figuras representam os cortes das diversas peças que serão blindadas nos carros Toyota Corolla e como será feito após a implantação e utilização do Software Powernest, que é uma biblioteca matemática destinada à otimização do aproveitamento de qualquer peça, fazendo uma integração de forma que o aproveitamento do corte das placas ocorra de forma completa.

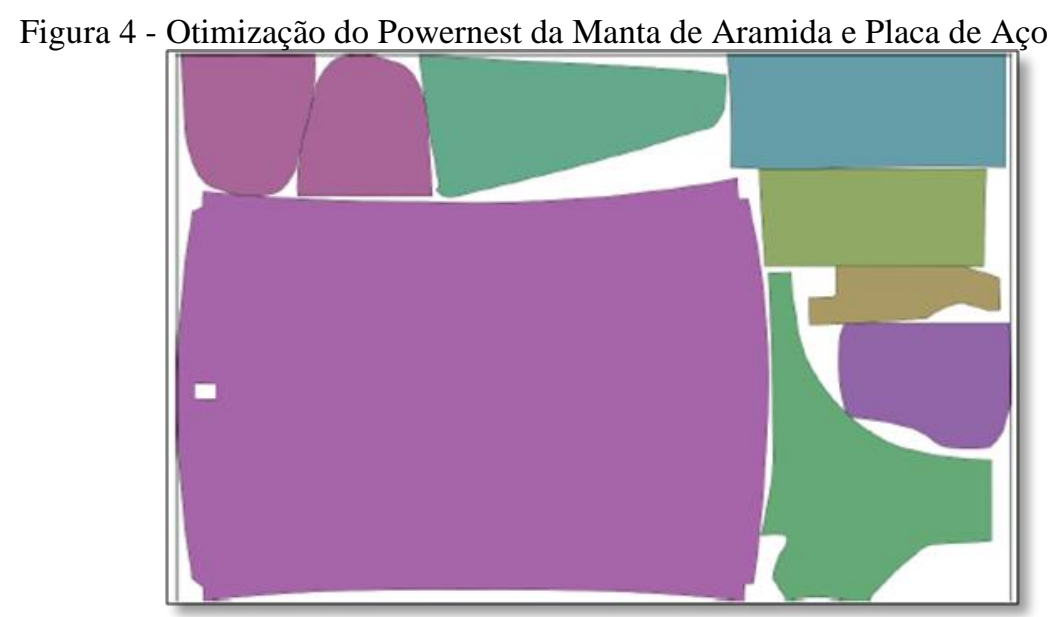

Fonte: Autores (2019) 
O Powernest é especialista no aproveitamento automático de formas complexas para o corte de materiais em chapas sendo a solução de aproveitamento automático flexível e extremamente eficaz, que permite reduzir sensivelmente os custos de matéria-prima e melhorar a produtividade, pois apresenta os planos de corte otimizados e a ordem de corte das peças prontas para a produção de blindagem do veículo, conforme é possível observar na figura 5;

Figura 5 - Otimização do Powernest da Manta de Aramida e Placa de Aço - Placa 2

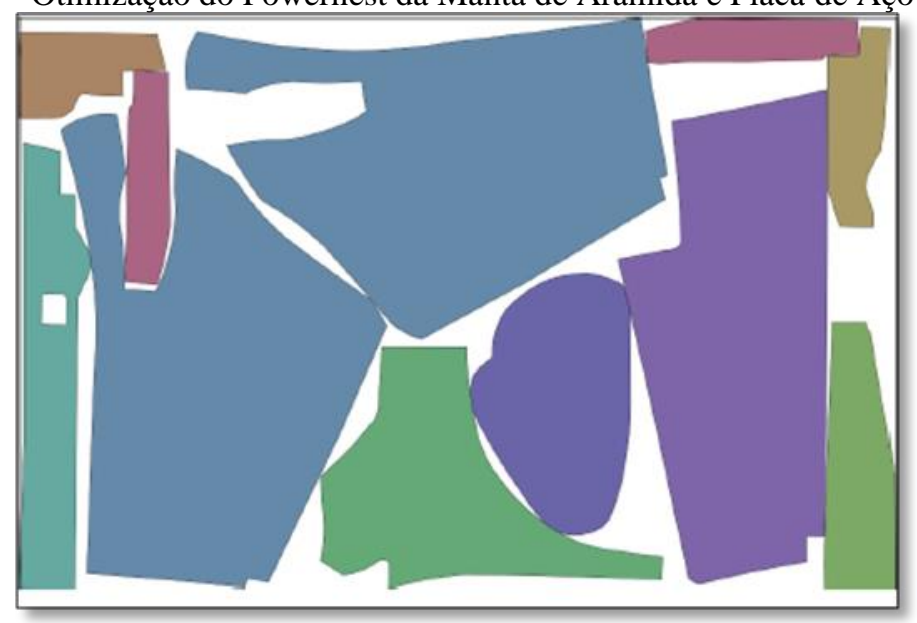

Fonte: Autores (2019)

Como pode-se observar na figura 6, por meio da rotação das peças segundo qualquer ângulo, posicionamento das peças e das prioridades de colocação, compactação de colocações existentes, otimização do espaçamento entre as peças de uma colocação existente, colocação de peças nos espaços livres a níveis.

Figura 6 - Otimização do Powernest da Manta de Aramida e Placa de Aço - Placa 3

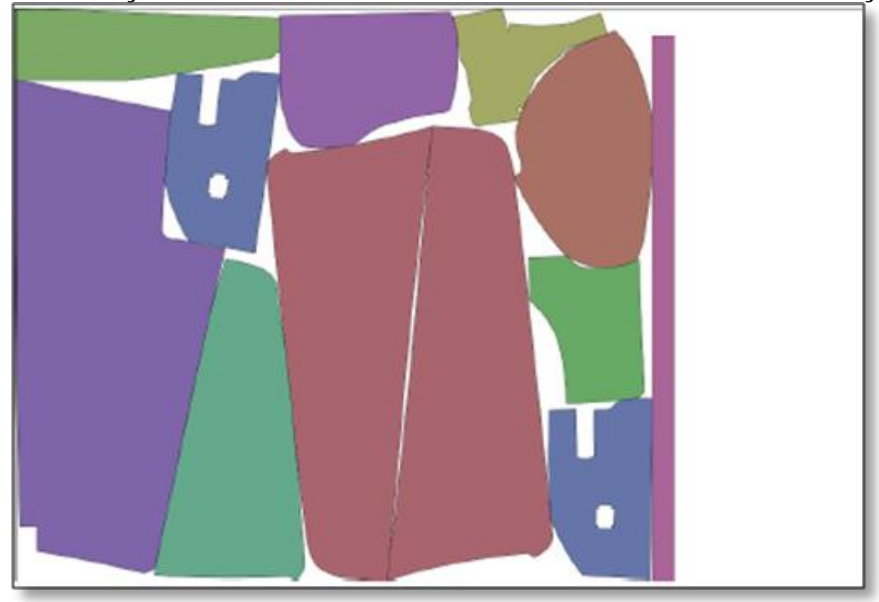

Fonte: Autores (2019) 
O aperfeiçoamento e otimização que se consegue com a utilização do software Powernest na modelagem que antes era realizada somente com a utilização do AutoCAD, resultou na racionalização dos formatos de chapa combinada ao desempenho, pois o software de aproveitamento permitiu diminuir a taxa de perdas.

A partir de Agosto/2019 iniciou-se a aplicação da solução proposta, e assim elaborou-se uma nova folha de verificação, conforme mostra a tabela 6 , constatando a otimização na produção com a implantação da padronização do processo e com a etapa dos moldes já automatizada.

Tabela 6 - Nova Folha de Verificação Ago/19 - Out/19

\begin{tabular}{|c|c|}
\hline \multicolumn{2}{|c|}{$\begin{array}{c}\text { Folha de Verificação do Toyota Corolla } \\
\text { Consolidado Ago/19 - Out/19 }\end{array}$} \\
\hline Erro operacional no processo de corte & 1 \\
\hline Erro operacional no processo de molde & 2 \\
\hline Falta de padronização & 0 \\
\hline Erro operacional no processo de marcação & 1 \\
\hline Perda de manta aramida & 2 \\
\hline Perda de placa aço & 2 \\
\hline Outras causas & 5 \\
\hline TOTAL & $\mathbf{1 3}$ \\
\hline
\end{tabular}

Fonte: Autores (2019)

Com os novos resultados obtidos através da atual Folha de Verificação, construiu-se um novo Gráfico de Pareto, conforme o gráfico 3, mostrando o resultado após a otimização;

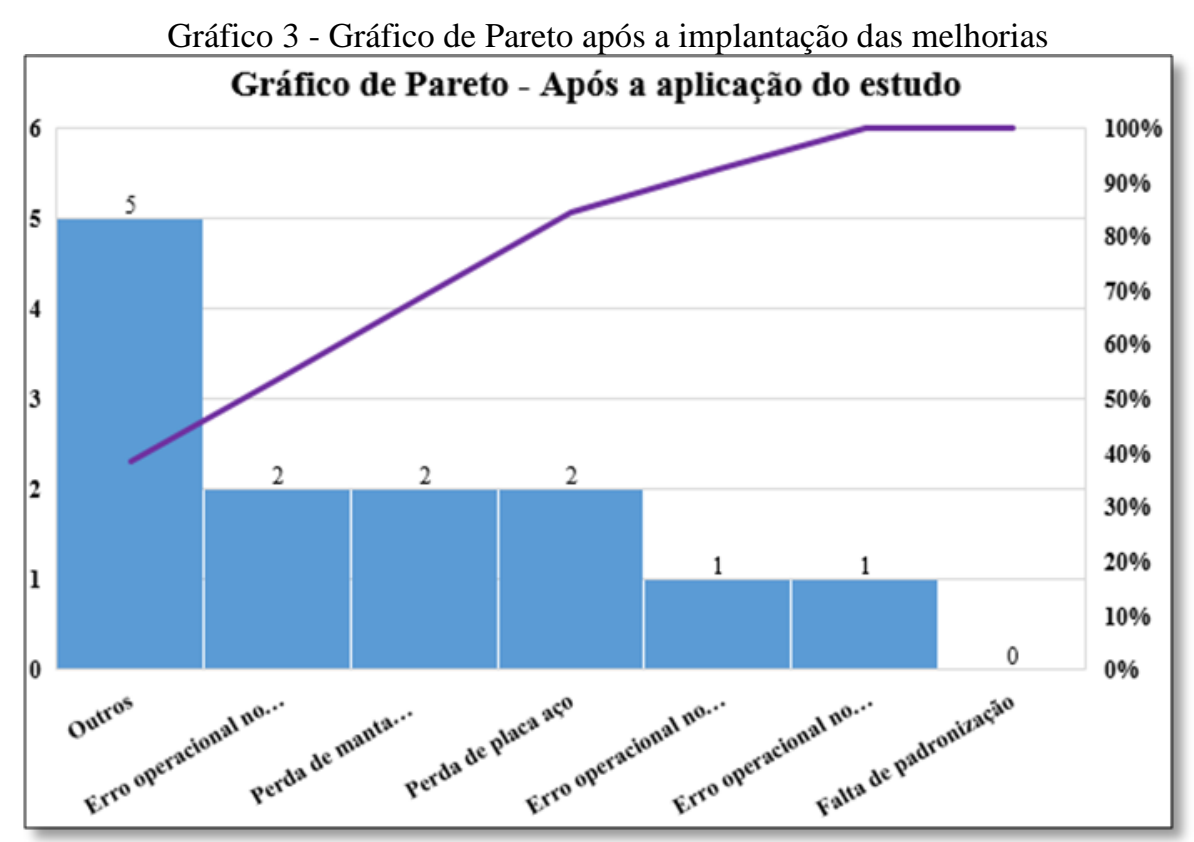

Fonte: Autores (2019) 
Na tabela 7, foi feita uma comparação da blindagem realizada em uma mesma quantidade de automóveis no período que vai de agosto a outubro de 2018 com o mesmo período em 2019, após a padronização e otimização do processo, onde é possível verificar a real diminuição das perdas e do prejuízo para a empresa.

Tabela 7 - Comparação Ago/Out - 2018 e 2019

\begin{tabular}{|c|c|c|c|c|c|}
\hline \multicolumn{6}{|c|}{2018 - Ago/Out } \\
\hline Mês/Ano & Quant. de Carros & Quant. de Placas & $\mathrm{m}^{2}$ da Manta & $\mathrm{m}^{2}$ do Aço & Valor Total \\
\hline ago/18 & 4 & 40 & $\mathrm{R} \$ 25.280,00$ & $\mathrm{R} \$ \quad 46.016,00$ & $\mathrm{R} \$ \quad 71.296,00$ \\
\hline set/18 & 5 & 50 & $\mathrm{R} \$ 31.600,00$ & $\mathrm{R} \$ \quad \mathbf{5 7 . 5 2 0 , 0 0}$ & $\mathrm{R} \$ \quad 89.120,00$ \\
\hline out $/ 18$ & 6 & 60 & $\mathrm{R} \$ 37.920,00$ & $\mathrm{R} \$ \quad 69.024,00$ & $\mathrm{R} \$ 106.944,00$ \\
\hline Total Obtido & 15 & 150 & R\$ $94.800,00$ & RS $172.560,00$ & R\$ $267.360,00$ \\
\hline
\end{tabular}

\begin{tabular}{|c|c|c|c|c|c|c|}
\hline \multicolumn{7}{|c|}{$\mathbf{2 0 1 9}$ - Ago/Out } \\
\hline Mês/Ano & Quant. de Carros & Quant. de Placas & $\mathbf{m}^{\mathbf{2}}$ da Manta & $\mathbf{m}^{\mathbf{2}}$ do Aço & Valor Total \\
\hline ago/19 & 4 & 24 & $\mathrm{R} \$ 15.168,00$ & $\mathrm{R} \$ 27.608,00$ & $\mathrm{R} \$$ & $42.776,00$ \\
\hline set/19 & 5 & 30 & $\mathrm{R} \$ 18.960,00$ & $\mathrm{R} \$ 34.510,00$ & $\mathrm{R} \$$ & $53.470,00$ \\
\hline out/19 & 6 & 36 & $\mathrm{R} \$ 22.752,00$ & $\mathrm{R} \$ 41.412,00$ & $\mathrm{R} \$$ & $64.164,00$ \\
\hline Total Obtido & $\mathbf{1 5}$ & $\mathbf{7 2}$ & $\mathrm{R} \$ \mathbf{5 6 . 8 8 0 , 0 0}$ & $\mathrm{R} \$ \mathbf{1 0 3 . 5 3 0 , 0 0}$ & $\mathrm{R} \$ \mathbf{1 6 0 . 4 1 0 , 0 0}$ \\
\hline
\end{tabular}

Na tabela 8, pode-se observar os resultados esperados para o ano de 2020;

Tabela 8 - Tabela de Custos Esperados 2019/2020

\begin{tabular}{|c|c|c|c|c|c|c|c|}
\hline \multicolumn{8}{|c|}{ Tabela de Custo de Produção Anual - Após do Estudo de Caso } \\
\hline Mês/Ano & Quant. de Carros & Quant. De Placas & $\mathrm{m}^{2}$ da Manta & & ${ }^{2}$ do Aço & & lor Total \\
\hline nov/19 & 7 & 42 & $\mathrm{R} \$ 26.544,00$ & R\$ & $48.314,00$ & $\mathrm{R} \$$ & $74.858,00$ \\
\hline dez/19 & 6 & 36 & R\$ $22.752,00$ & RS & $41.412,00$ & $\mathrm{R} \$$ & $64.164,00$ \\
\hline jan/20 & 8 & 48 & R\$ $30.336,00$ & RS & $55.216,00$ & $\mathrm{R} \$$ & $85.552,00$ \\
\hline fev $/ 20$ & 15 & 90 & R\$ $\quad 56.880,00$ & R\$ & $103.530,00$ & $\mathrm{R} \$$ & $160.410,00$ \\
\hline $\mathrm{mar} / 20$ & 9 & 54 & R\$ $34.128,00$ & $\mathrm{RS}$ & $62.118,00$ & $\mathrm{R} \$$ & $96.246,00$ \\
\hline abr $/ 20$ & 9 & 54 & $\mathrm{R} \$ \quad 34.128,00$ & $\mathrm{RS}$ & $62.118,00$ & $\mathrm{R} \$$ & $96.246,00$ \\
\hline $\mathrm{mai} / \mathbf{2 0}$ & 11 & 66 & R\$ $41.712,00$ & RS & $75.922,00$ & $\mathrm{R} \$$ & $117.634,00$ \\
\hline jun/20 & 6 & 36 & $\mathrm{R} \$ \quad 22.752,00$ & RS & $41.412,00$ & $\mathrm{R} \$$ & $64.164,00$ \\
\hline jul/20 & 8 & 48 & R\$ $30.336,00$ & $\mathrm{RS}$ & $55.216,00$ & $\mathrm{R} \$$ & $85.552,00$ \\
\hline ago/20 & 6 & 36 & R\$ $22.752,00$ & $\mathrm{R} \$$ & $41.412,00$ & $\mathrm{R} \$$ & $64.164,00$ \\
\hline Total Esperado & 100 & 510 & RS $322.320,00$ & RS & $586.670,00$ & RS & $908.990,00$ \\
\hline
\end{tabular}

Fonte: Autores (2019)

A tabela foi montada a partir de dados já conhecidos da empresa PCB, a expectativa para o período de novembro de 2019 a agosto de 2020, é que através do processo de padronização implantado obtenha-se um aumento significativo na lucratividade da empresa. 


\section{Considerações Finais}

Com a utilização do software pôde-se ter um melhor aproveitamento da matéria prima, com a impressão realizada em uma lona com medidas igual à Manta de Aramida e a Placa de Aço, com os moldes já em sua disposição há menos perda.

Todo este processo de melhoria, só foi possível devido ao uso das técnicas de BPM, Mapeamento de Processo, Indicadores de Processo e Ferramentas da Qualidade, que utilizadas em conjunto propiciou a diminuição das perdas e a eliminação dos desperdícios, otimizando o processo e gerando lucratividade para a empresa PCB.

Ao final do trabalho, após as aplicações das técnicas citadas, foram encontrados resultados satisfatórios, ocorrendo o cumprimento de todos os objetivos proposto para este estudo. Por meio disso, foi implantada a padronização de corte com o auxilio do software Powernest que otimizou os moldes para melhoria do processo e consequentemente a diminuição de perda de matéria prima, reduzindo assim os custos desnecessários que eram gerados devido as perdas. 
Referências Bibliográficas

ABPMP - Association of Business Process Management Professionals. BPM CBOK BUSINESS PROCESS MANAGEMENT: common body of knowledge. Chicago: ABPMP, 2009.

BORTOLUZZI, Sandro et. al. MAPEAMENTO DOS INDICADORES DE DESEMPENHO ORGANIZACIONAL EM PESQUISAS DA ÁREA DE ADMINISTRAÇÃO, CIÊNCIAS CONTÁBEIS E TURISMO NO PERÍODO DE 2000 A 2008. R.Adm., São Paulo, v.46, n.4, p.373-391, 2011.

CAMPOS, Vicente. QUALIDADE TOTAL - PADRONIZAÇÃO DE EMPRESAS - $\mathbf{2}^{\mathrm{a}}$ Ed, 2014.

COSTA, Marly et. al. INDÚSTRIA DE CARTUCHO DE TONER SOB A ÓTICA DA REMANUFATURA: ESTUDO DE CASO DE UM PROCESSO DE MELHORIA. Produção, v. 16, n. 1, p. 100-110, Jan./Abr. 2006.

CUNHA, Alex. MAPEAMENTO DE PROCESSOS ORGANIZACIONAIS NA UnB: Caso de Centro de Documentação da UnB. Brasília, 2012.

FONSECA, Erika et. al. A INFLUÊNCIA DAS FERRAMENTAS DA QUALIDADE NA PRODUÇÃO DE EMBALAGENS SECUNDÁRIAS. ENCONTRO NACIONAL DE ENGENHARIA DE PRODUÇÃO (ENEGEP), 2018.

FRANCO, Matheus et. al. COLAÇÃO DE GRAU: BENEFÍCIOS OBTIDOS POR MEIO DO REDESENHO DE PROCESSOS. ENCONTRO NACIONAL DE ENGENHARIA DE PRODUÇÃO (ENEGEP), 2018.

LADEIRA, Marcelo et. al. GESTÃO DE PROCESSOS, INDICADORES ANALÍTICOS E IMPACTOS SOBRE O DESEMPENHO COMPETITIVO EM GRANDES E MÉDIAS EMPRESAS BRASILEIRAS DOS SETORES DA INDÚSTRIA E DE SERVIÇOS. Gest. Prod., São Carlos, v. 19, n. 2, p. 389-404, 2012.

MEDEIROS, I.; GUTIERREZ, R. O MAPEAMENTO DE PROCESSOS COMO FATOR DE MELHORIA DA GESTÃO ACADÊMICA DE UMA UNIVERSIDADE FEDERAL. Congresso Nacional de Excelência em Gestão, 2015. 
MILAN, G.; SOSO, F. BPM - BUSINESS PROCESS MANAGEMENT COMO PRÁTICA DE GESTÃO EM UMA EMPRESA METALÚRGICA COM ESTRATÉGIA DE PRODUÇÃO ETO - ENGINEER-TO-ORDER. Revista Gestão Industrial, Paraná, v. 08, n. 02: p. 164-185, 2012.

MILNITZ, Diego et. al. O GERENCIAMENTO DE PROCESSOS DE NEGÓCIO (BPM) NOS PROCESSOS LOGÍSTICOS - UMA REVISÃO DE LITERATURA. Exacta - EP, São Paulo, v. 14, n. 3, p. 419-430, 2016.

NASCIMENTO, Stenio. UTILIZAÇÃO DA MODELAGEM BPMN PARA MAPEAR O PROCESSO DE UMA LINHA DE PRODUÇÃO EM UMA INDÚSTRIA DE ALIMENTOS. ENCONTRO NACIONAL DE ENGENHARIA DE PRODUÇÃO (ENEGEP), 2018.

OLIVEIRA, Uarlen et. al. BPMN E ORGANIZAÇÃO CELULAR DE EQUIPES NA IMPLANTAÇÃO DE GERENCIAMENTO POR PROCESSOS EM EMPRESA SIDERÚRGICA. ENCONTRO NACIONAL DE ENGENHARIA DE PRODUÇÃO (ENEGEP), 2018.

ZANIN, Antonio et. al. DEFINIÇÃO DE PAINEL DE INDICADORES DE DESEMPRENHO PARA INSTITUIÇÕES COMUNITÁRIAS DE ENSINO SUPERIOR. Revista GUAL, Florianópolis, v. 8, n. 2, p. 01-27, 2015. 